\title{
CONGENITAL HYDROCEPHALUS IN MONOVULAR TWINS
}

\author{
BY \\ V. GELLMAN \\ From the Department of Pediatrics, University of Illinois, Chicago, U.S.A.
}

(RECEIVED FOR PUBLICATION JANUARY 2, 1959)

Congenital hydrocephalus in monovular twins is very rare. Borle (1953) published an extensive review of the cases reported since 1899 which purported to demonstrate the familial character of hydrocephalus. Her work covered the English, American and European publications and included seven pairs of monovular twins with hydrocephalus occurring in each twin (Booth, 1911; Siemens, 1924; Weise, 1935, de Lange, 1936; Fischer, 1942, Polman, 1950; Klein, Bersot and Borle, 1953). However, the presence of hydrocephalus was not definitely proven in some of these cases.

For example, in Booth's case the evidence for hydrocephalus is scant. His twins were $2 \frac{1}{2}$-year-old female pyopages united in the sacrococcygeal region. They were bright and intelligent, but the shape of their heads was 'peculiar'. They had bulging foreheads and flat vertices. Booth suggested that the children had almost a hydrocephalic appearance but he gave no further evidence to support his impression.

Siemens described identical twin brothers aged 4 months. Their head circumferences were $45 \cdot 5 \mathrm{~cm}$. and $45 \mathrm{~cm}$. respectively. Both had cerebral atrophy and severe external hydrocephalus of unknown aetiology. The author postulated a hereditary aetiological factor.

The twins described by Weise survived until adult life. Their head circumferences were $58 \mathrm{~cm}$. and $57 \mathrm{~cm}$. respectively. Each had a mild degree of hydrocephalus. Both were retarded and in addition one had syringomyelia.

De Lange (1936) described twins aged 8 months with head circumferences of $46 \mathrm{~cm}$. and $45 \mathrm{~cm}$. Their skulls were natiform from birth and they both had a mild degree of rickets. Their anterior fontanelles were bulging and in one the cerebral spinal fluid pressure was increased. The author believed that hydrocephalus was the cause of the skull deformity.

The cases reported by Fischer (1942) were probably not congenital hydrocephalics. Their head circumferences at birth were $32 \mathrm{~cm}$. and $33 \mathrm{~cm}$.
Enlargement of the head was first noticed in early childhood. The twins both died and at autopsy definite signs of meningitis were seen.

Polman (1950) made only a brief reference to a pair of hydrocephalic twins who were 'presumably monozygotic'. No further details were given.

The patients reported by Borle (1953) herself were a pair of male monovular twins. Head enlargement became apparent in each twin sometime between 2 and 6 months. There was no accompanying illness, but convulsions began at about 8 months. At 2 years their head circumferences were 53 and $54 \mathrm{~cm}$. respectively. The anterior fontanelles were still open and radiographs of the skull showed changes typical of hydrocephalus. Both boys were mentally retarded. They began to walk at 2 years and to talk at 4 years. There was in addition another hydrocephalic sibling.

In 1956 Cohn, Weinburg and Rockaway published a report on the birth of achondroplastic monovular twins with hydrocephalus, who died soon after birth. The mother was subsequently delivered of a third hydrocephalic achondroplastic infant. In the case of the twins, the hydrocephalus was caused by bony hypertrophy in the region of the foramen magnum obliterating the subdural and epidural space. One can assume, therefore, that the hydrocephalus was not primarily of genetic origin but secondary to the changes in the skull due to achondroplasia.

Of the eight cases mentioned above, the hydrocephalus in two pairs of twins was secondary to some other aetiological factor (Fischer, 1942; Cohn et al., 1956). This leaves six cases which may support the theory that some cases of congenital hydrocephalus are genetically determined. A seventh case is presented.

Case Report. S. and N. were 5-year-old identical twin boys who were delivered at term after a normal pregnancy by caesarian section because of interlocking heads. The boys weighed between 8 and $9 \mathrm{lb}$. at birth. They presented no feeding problems, sat at 4 months, walked at 18 months, and had no serious illnesses. However, at 
5 years they both developed stomatitis and were taken to their physician. He was impressed by the large size of their heads and their obvious mental retardation, and referred them to hospital.

The parents were Palestinian Arabs. The father was 60 and the mother 33 . They were both healthy and of average intelligence, although their knowledge of English was limited. The sister and brother, aged 4 and 3 years respectively, were normal. A sibling born before the twins had died at 3 days of unknown cause. According to the mother this infant had had a normal head size.

S. and N. were physically well-developed boys. S. was $129 \mathrm{~cm}$. tall and weighed $64 \mathrm{lb}$., and N. was $129 \mathrm{~cm}$. tall and weighed $63 \frac{1}{2} \mathrm{lb}$. Their head circumferences were 55 and $56 \mathrm{~cm}$. respectively. Apart from their obviously large heads, vacant, open-mouthed expression (see Fig. 1), and slowness of affect, there were no abnormal physical findings on examination. They were quiet, bashful boys who seemed to lack animation and who were extremely dependent on their parents. During one week in the ward they neither talked to each other nor played with the other children, but cried constantly for their mother.

Relevant laboratory investigations are listed in Table 1.

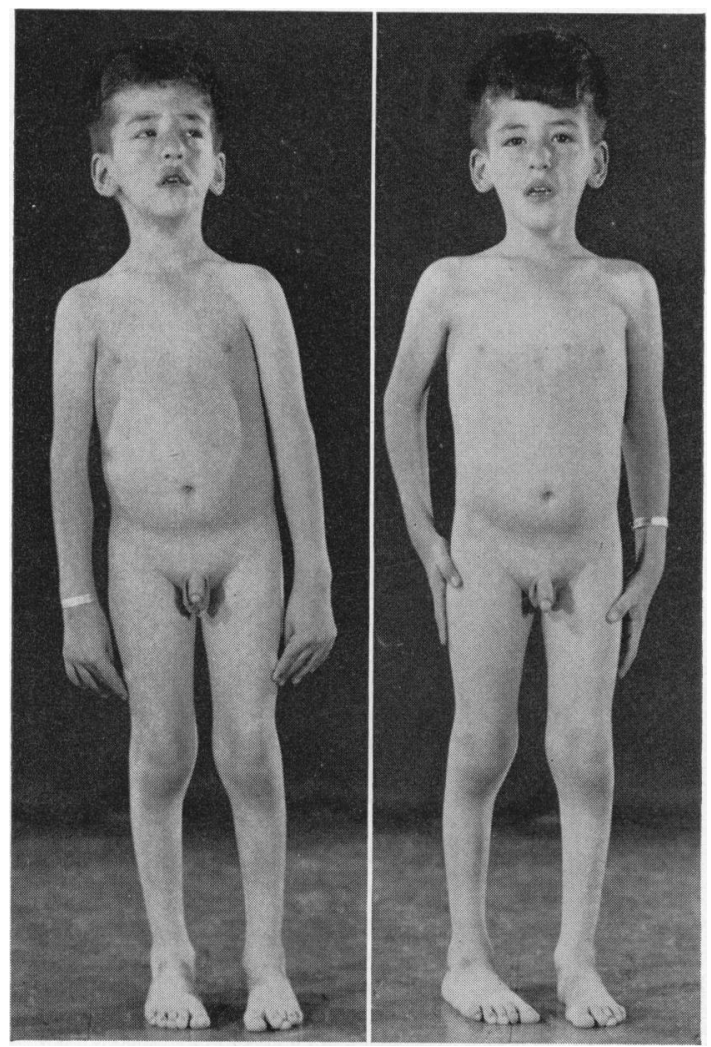

FIG. 1.-N.J. and S.J., aged 5 years. Both children have large heads and vacant expressions.
TABLE 1

LABORATORY INVESTIGATIONS

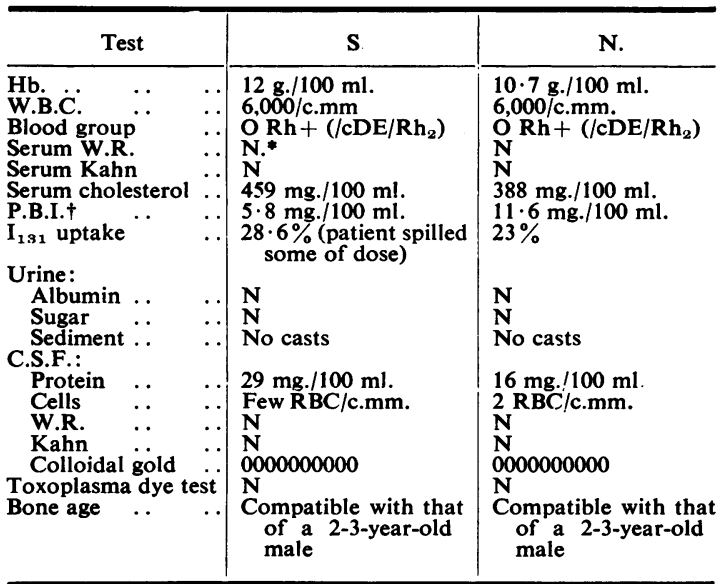

$* \mathbf{N}=$ negative.

$\dagger$ Blood samples were taken soon after iodine mouth washes.

Electroencephalograms in both patients showed 14 and 6 per second positive spike seizure activity in both hemispheres.

Pneumoencephalography in both patients showed an increase in the size of the skull. In the case of S., the occipito-frontal diameter was $20.5 \mathrm{~cm}$., the biparietal diameter was $17.5 \mathrm{~cm}$., and the height $16.5 \mathrm{~cm}$. In the case of $\mathrm{N}$., the occipito-frontal diameter was $21.5 \mathrm{~cm}$., the biparietal diameter was $17.5 \mathrm{~cm}$., and the height $18 \mathrm{~cm}$. There was moderate symmetrical distension of the ventricular system in both patients. These findings are compatible with hydrocephalus. The dorsum sellae and sutures were normal.

Psychological testing was attempted in both children but was difficult because of lack of co-operation. N. was described as 'untestable' but it was the examiner's impression that he was retarded. The test on S. revealed intellectual limitation of a degree which could not be accurately determined.

\section{Comment}

Occasionally it is possible to find an exogenous factor such as syphilis, meningitis, toxoplasmosis, or obstetrical trauma as the underlying cause of congenital hydrocephalus. Frequently, however, no organic cause can be detected. In such cases the question of a genetic factor arises, particularly when the condition occurs in monovular twins. The fact that there is a strong familial tendency to congenital hydrocephalus would also support the genetic theory.

In the case of the twins presented above, toxic factors and obstetrical trauma were eliminated. The history and investigations suggested that this was an authentic case of congenital hydrocephalus in monovular twins. It is postulated that the hydro- 
cephalus was of genetic aetiology. In the presence of mental retardation the high cholesterol values and the retarded bone age are suggestive of hypothyroidism. However, the patients were of normal stature and other tests of thyroid function did not confirm this diagnosis.

\section{Summary}

The case histories are reported of identical twins with congenital hydrocephalus and mental retardation. The rarity of such cases is demonstrated by a brief review of the publications on this subject. The cases are presented in support of the theory that some examples of congenital hydrocephalus may be genetically determined.

I wish to express my thanks to Dr. Heyworth $\mathbf{N}$. Sanford for permission to publish the cases, and to Dr. Ira M. Rosenthal for helpful criticism of the paper.

\section{REFERENCES}

Booth, J. A. (1911). Brit. med. J., 2, 653.

Borle, A (1953). J. Génét hum 2, 157.

Cohn, S., Weinberg, A. and Rockaway, F. (1956). Amer. J. Obstet. Gynec., 72, 1346

De Lange, C. (1936). Arch. Dis. Childh., 11, 39.

Fischer, H. (1942). Z. ges. Neurol. Psychiat., 174, 264.

Klein, D., Bersot, H. and Borle, A. (1953). Confin. neurol., 13, 158

Polman, A. (1950). Genetica 25, 29.

Siemens, H. W. (1924). Die Źwillings pathologie, p. 82. J. Springer Berlin.

Weise, G. (1935). Arch. Psychiat. Nervenkr., 103, 191. 